\title{
Spontaneous Currents in a Bosonic Ring
}

\author{
D. MAKIEŁA AND M.M. MAŚKA* \\ Department of Theoretical Physics, Institute of Physics, University of Silesia, \\ Uniwersytecka 4, 40-007 Katowice, Poland
}

\begin{abstract}
Nonequilibrium dynamics of non-interacting bosons in a one-dimensional ring-shaped lattice is studied by means of the kinetic Monte Carlo method. The system is approximated by the classical XY model (the kinetic term is neglected) and then the simulations are performed for the planar classical spins. We study the dynamics that follows a finite-time quench to zero temperature. If the quench is slow enough the system can equilibrate and finally reaches the ground state with uniform spin alignment. However, we show that if the quench is faster than the relaxation rate, the system can get locked in a current-carrying metastable state characterized by a nonzero winding number. We analyze how the zero-temperature state depends on the quench rate.
\end{abstract}

DOI: 10.12693/APhysPolA.130.569

PACS/topics: 03.75.Lm, 03.75.Kk, 64.60.Ht

\section{Introduction}

It has been predicted many years ago that small metallic rings may have a symmetry-breaking ground state with spontaneous orbital currents, i.e., currents occurring in the absence of applied magnetic field or power sources [1]. Similar behaviour was also expected as a result of the parity effect in superconducting nanorings [2] or chiral tunneling in carbon nanotubes [3]. Rotating ground state was predicted in a one-dimensional spinpolarized gas composed of an even number of fermionic atoms interacting via attractive $p$-wave interactions and confined to a mesoscopic ring [4]. In 1977 Bulaevskii et al. proposed that current can flow in the ground state of a superconducting ring with $\pi$-junction [5]. Such currents have recently been observed in superconducting networks [6]. Realization of this idea in superfluid fermionic cold gases in a ring-shaped trap has been proposed [7].

In this paper we demonstrate that a similar phenomenon can be expected also in a system of free noninteracting bosons in a one-dimensional ring. The difference with respect to the mentioned above examples is that in our case the current flows in a metastable state, not in the ground state. We show, however, that the system can be easily trapped in such a state by a sufficiently fast temperature quench that leads to a nonuniform symmetry breaking.

Non-uniform symmetry breaking was studied by Kibble [8], who has shown that the cooling down of the early universe resulted in independent symmetry breakings in distant regions. This, in turn, led to the formation of topological defects such as point-like monopoles, linear cosmic strings, or planar domain walls. Structures analogous to cosmic strings exist in many condensed matter systems [9]. Some examples of these defects are magnetic

*corresponding author; e-mail: maciej.maska@phys.us.edu.pl flux tubes in superconductors, vortices in superfluids, and certain defects in liquid crystals. The theory containing a complex field $\phi$ constitutes the simplest example which has string analogues. The mean value of $\phi$ above the critical temperature is equal to zero. Below this temperature, $\phi$ acquires a non-zero value that causes symmetry breaking as a consequence of a phase transition. One of the examples of such systems is superfluid helium-4. At low temperature a significant part of atoms of superfluid helium- 4 occupies a single quantum state. Then, $\phi$ is proportional to the wave function. A vortex is a topological defect around which the phase of $\phi$ changes by $2 \pi$. There occurs quantized circulation around the vortex because the superfluid velocity is proportional to the phase gradient. In the case of superconductors, the quantized quantity is the magnetic flux carried by the vortex.

One of the first experiments in which a rapid transition led to the formation of linear defects was done on nematic liquid crystals formed of rod-shaped molecules [10]. Another example is the experiments performed on superfluid helium-3 at the temperature around $2 \mathrm{mK}$. Helium- 3 reacts with slow neutrons as ${ }_{0}^{1} n+{ }_{2}^{3} H e \rightarrow{ }_{1}^{1} p+{ }_{1}^{3} H$, releasing $764 \mathrm{keV}$ of energy. In experiments where this reaction was used the neutron absorption heated up a small area of superfluid helium-3 above the critical temperature [11, 12]. Fast cooling of this area below the transition temperature led to the formation of vortices. In an experiment performed at CNRS Center for Research on Ultra-Low Temperatures in Grenoble, France [11], the total energy released after neutron absorption was investigated. It was found that observed energy deficit can be explained by the formation of vortices.

Several experiments were carried out also on superconductors. Some of them involved thin films of high temperature superconductors, first heated above the critical temperature, and then cooled back through the phase transition. The generated defects, which are tubes of quantized magnetic flux passing through the film in one direction or the other, are called fluxons and antifluxons [13]. Researchers measured the net defect number 
$\Delta N=N_{+}-N_{-}$where $N_{+}$and $N_{-}$are the numbers of fluxons and antifluxons, respectively. Kirtley and others performed experiments on thin-film superconducting rings [14].

Interesting experiments were performed also with the Josephson junctions. Monaco and collaborators used the annular Josephson junctions [15]. The rapid cooling generated a flux in the system. Then, the dependence of the probability of catching fluxon on the quench rate was measured. Cold atomic gases are another type of system in which there are vortices. Their advantage is high purity and theoretical simplicity [16].

The experiments mentioned above are evidences of the fact that a rapid phase transition leads to the creation of topological defects. Below we demonstrate how such a process can be quantitatively studied in a simple model of noninteracting itinerant bosons in a one-dimensional lattice with periodic boundary conditions.

\section{Model Hamiltonian}

We start with a Hamiltonian that describes the kinetic energy of noninteracting bosons, given by

$$
H=t \sum_{\langle i, j\rangle} b_{i}^{\dagger} b_{j},
$$

where $b_{i}\left(b_{i}^{\dagger}\right)$ is an operator that annihilates (creates) a boson in site $i, t$ is the hopping amplitude, $\langle i, j\rangle$ denotes summation over nearest neighbouring sites. Using the approximation

$$
b_{i} \rightarrow \sqrt{n_{i}} e^{i \theta_{i}}, \quad n_{i}=n=\mathrm{const},
$$

Hamiltonian (1) can be rewritten as the XY Hamiltonian

$$
H=J \sum_{\langle i, j\rangle} \cos \left(\theta_{i}-\theta_{j}\right),
$$

where $J=2 t n$. Approximation (2), where bosonic operators are replaced by $C$-numbers, neglects quantum fluctuations and generally is not valid. ${ }^{\dagger}$ However, with increasing $n$ the fluctuations become small. The Hamiltonian (3) is particularly justified if there are the Bose-Einstein condensates in all lattice sites. Such systems have been realized experimentally in cold atoms [18].

Complex variables $\theta_{i}$ in Eq. (3) can be interpreted as directions of planar spins with their dynamics governed by interaction $J \cos \left(\theta_{i}-\theta_{j}\right)$. At high temperature they are random. However, with decrease of temperature the interaction energy try to align the spins in the same direction. The one-dimensional XY model does not have a long range order at any finite temperature, so we can expect the fully polarized state only at $T=0$ (see Fig. 1a and $\mathrm{b}$ ).

The interaction energy is minimal if a given spin points in the same direction as its neighbours. If the system

\footnotetext{
${ }^{\dagger}$ Without approximations Hamiltonian (1) can be mapped onto the two-dimensional XY model [17].
}

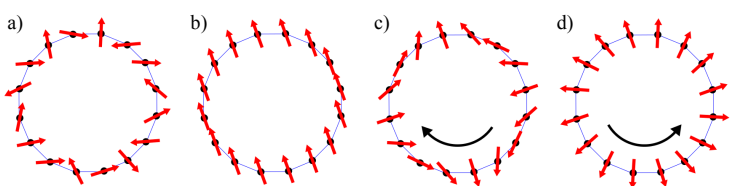

Fig. 1. Spin configurations in the one-dimensional ring-shaped lattice at high temperature (a) and at $T=$ 0 (b, c, and d). Part (b) shows configuration with $W_{N}=0$, whereas parts (c) and (d) configurations with $W_{N}=+1$ and $W_{N}=-1$, respectively.

is approaching the symmetry-breaking phase slowly, the spins can evolve almost adiabatically eventually reaching the global minimum of energy, i.e, the configuration presented in Fig. 1b. However, if the temperature quench is sufficiently fast, the system can end up in a local minimum of energy where spins are (almost) parallel only locally. Since the phase $\theta_{i}$ must be a single-valued function of position $i$, its total change along a closed path must be equal to $2 \pi W_{N}$, where $W_{N}$ is an integer. Then, all spin configurations can be classified by the value of $W_{N}$ and only the one with $W_{N}=0$ corresponds to the global minimum of energy. Since $W_{N}$ 's are integer, configurations with different $W_{N}$ cannot be continuously transformed one into another. $W_{N}$ is named a winding number and can formally be defined as:

$$
W_{N}=\frac{1}{2 \pi} \sum_{\langle i, j\rangle}\left(\theta_{i}-\theta_{j}\right) .
$$

Finite value of the winding number requires a non-zero gradient of the boson wave function and indicates the existence of a current flowing along the ring [19]. Examples of such configurations are presented in Fig. 1c and d.

The question we want to address in this paper is how the value of $W_{N}$ depends on the cooling rate. In the case of the second order phase transition the answer is suggested by the famous Kibble-Żurek hypothesis [8, 9].

\section{Simulations of the spin dynamics}

Here, we propose to perform a computer experiment to "measure" $W_{N}$ for different cooling rates. The most natural approach would be to use the Monte Carlo (MC) method to simulate the behaviour of the system during the temperature quench. The problem, however, is that in the standard MC approaches there is no relation between the number of MC steps and the real time. This relation is particularly disturbed close to a phase transition where the critical slowing down occurs. In order to overcome these difficulties we propose to use the kinetic Monte Carlo (KMC) method [20]. It is a method commonly used to study time dependence of processes occurring in nature. If these processes have known transition rates between different states, KMC allows one to determine the relation between the number of steps in the algorithm and the real time of the process. In the case of the XY model the transition time is defined by the inverse of the coupling $J$. 
The computer "experiments" are performed as follows: For a given number of lattice sites $N$ we chose an initial random high-temperature configuration of the spins. Then, the temperature $T(t)$ decreases linearly with time to zero. The quench time is defined by $\tau_{Q}$ :

$$
T(t)= \begin{cases}-T_{0} \frac{t}{\tau_{Q}} & \text { for } t \in\left[-\tau_{Q}, 0\right), \\ 0 & \text { for } t \in\left[0, t_{R}\right),\end{cases}
$$

where $T_{0} \gg J$ and time $t_{R}$ is sufficiently long to allow the system to relax to its local energy minimum after the temperature quench. The evolution of the system is defined by the following algorithm sketched in Fig. 2.

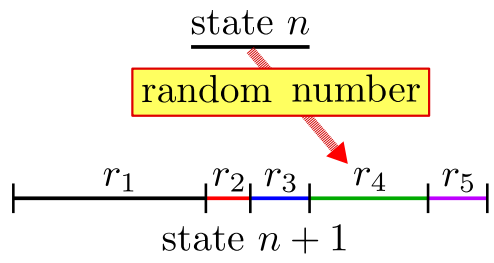

Fig. 2. Illustration of the kinetic Monte Carlo method. The probability that in a given MC step the system will evolve to state $i$ is proportional to $r_{i}$. See the text for detailed explanation.

At each step of simulation we must consider all possible ways of evolution to the next state. The corresponding transition probabilities $r_{i}$ are given by the change of energy $\Delta E$ between the states and the current temperature of the system, $r_{i} \propto \exp \left(-\Delta E_{i} / k_{B} T\right)$. Next, we compute the cumulative function $R_{i}=\sum_{n=1}^{i} r_{n}, \quad i=$ $1, \ldots, M$, where $M$ is the number of all the states that can be directly reached from the present state, and generate a random number $q$ from the uniform distribution $\left(0, R_{N}\right]$. We find the index $j$ satisfying inequality $R_{j-1}<q \leq R_{j}$. It means that the number $q$ is in the field $r_{j}$ (Fig. 2) and the system will go to state $j$. The main numerical difficulty in this method is that the number of possible transitions from a given state increases exponentially with the number of lattice sites.
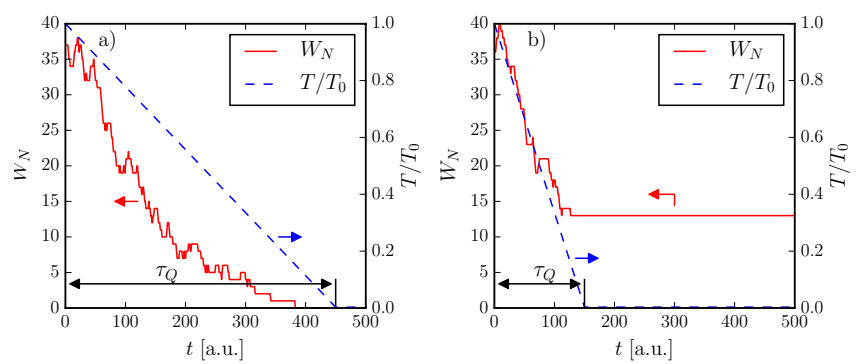

Fig. 3. The time dependence of the winding number $W_{N}$ for slow (a) and fast (b) cooling. The solid red line shows the time evolution of $W_{N}$, whereas the dashed blue line shows the temperature. The black horizontal arrow indicates the value of the cooling time $\tau_{Q}$ [Eq. (5)]. Part (b) illustrates a situation where after the quench the system remains in a state with non-zero winding number.
After the quench the system evolves to its final configuration, and then we "measure" the winding number. Figure $3 \mathrm{a}$ and $\mathrm{b}$ show the evolution in the case of slow and fast temperature quench, respectively.

The results show that when the cooling is slow the system is able to maintain its quasi adiabatic evolution and eventually ends up in the state corresponding to the global minimum of energy with $W_{N}=0$. On the other hand, if the quench is faster than the relaxation rate, the system can get locked in a metastable current-carrying state with non-zero $W_{N}$. Detailed analysis of the results show that the dependence of the average value of $W_{N}$ on the quench time $\tau_{Q}$ and on the system size $N$ is in agreement with predictions of the Kibble-Żurek hypothesis.

\section{Conclusions}

We have shown that the temperature quench in a bosonic ring can produce spontaneous currents. The most natural system where such currents could be observed is a ring-shaped optical lattice with the Bose-Einstein condensates in each lattice site $[18,21,22]$. It is, however, possible that similar effects may be realized by temperature quench also in other systems described by the onedimensional XY model, e.g., granular superconducting rings or rings of the Josephson junctions [23].

\section{Acknowledgments}

This work was supported by the Polish National Science Centre (NCN) under grant DEC2013/11/B/ST3/00824.

\section{References}

[1] D. Wohlleben, M. Esser, P. Freche, E. Zipper, M. Szopa, Phys. Rev. Lett. 66, 3191 (1991); M. Rasolt, F. Perrot, Phys. Rev. Lett. 69, 2563 (1992); M.Y. Choi, Phys. Rev. Lett. 71, 2987 (1993).

[2] S.V. Sharov, A.D. Zaikin, Physica E 29, 360 (2005).

[3] A.V. Parafilo, I.V. Krive, E.N. Bogachek, U. Landman, R.I. Shekhter, M. Jonson, Phys. Rev. B 83, 045427 (2011).

[4] M.D. Girardeau, E.M. Wright, Phys. Rev. Lett. 100, 200403 (2008).

[5] L.N. Bulaevskii, A.A. Sobyanin, V.V. Kuzii, Zh. Eksper. Teor. Fiz. 25, 314 (1977).

[6] S.M. Frolov, M.J.A. Stoutimore, T.A. Crane, D.J. Van Harlingen, V.A. Oboznov, V.V. Ryazanov, A. Ruosi, C. Granata, M. Russo, Nat. Phys. 4, 32 (2008).

[7] T. Kashimura, S. Tsuchiya, Y. Ohashi, Phys. Rev. A 84, 013609 (2011).

[8] T.W.B. Kibble, J. Phys. A Math. Gen. 9, 1387 (1976); T.W.B. Kibble, Phys. Rep. 67, 183 (1980).

[9] W.H. Żurek, Nature 317, 505 (1985).

[10] I. Chuang, R. Durrer, N. Turok, B. Yurke, Science 251, 1336 (1991). 
[11] C. Büerle, Yu.M. Bunkov, S.N. Fisher, H. Godfrin, G.R. Pickett, Nature 382, 332 (1996).

[12] V.M.H. Ruutu, V.B. Eltsov, A.J. Gill, T.W.B. Kibble, M. Krusius, Yu.G. Makhlin, B. Plaçais, G.E. Volovik, Wen Xu, Nature 382, 334 (1996).

[13] A. Maniv, E. Polturak, G. Koren, Phys. Rev. Lett. 91, 197001 (2003)

[14] J.R. Kirtley, C.C. Tsuei, F. Tafuri, Phys. Rev. Lett. 90, 257001 (2003).

[15] R. Monaco, M. Aaroe, J. Mygind, R.J. Rivers, V.P. Koshelets, Phys. Rev. B 74, 144513 (2006).

[16] L. Mathey, A. Polkovnikov, A.H. Castro Neto, Europhys. Lett. 81, 10008 (2008); L.E. Sadler, J.M. Higbie, S.R. Leslie, M. Vengalattore, D.M. Stamper-Kurn, Nature 443, 312 (2006); D.R. Scherer, C.N. Weiler, T.W. Neely, B.P. Anderson, Phys. Rev. Lett. 98, 110402 (2007).

[17] M.A. Cazalilla, R. Citro, T. Giamarchi, E. Orignac, M. Rigol, Rev. Mod. Phys. 83, 1405 (2011).
[18] D.R. Scherer, C.N. Weiler, T.W. Neely, B.P. Anderson, Phys. Rev. Lett. 98, 110402 (2007); K. Henderson, C. Ryu, C. MacCormick, M.G. Boshier, New J. Phys. 11, 043030 (2009).

[19] R. Monaco, J. Mygind, R.J. Rivers, V.P. Koshelets, Phys. Rev. B 80, 180501(R) (2009).

[20] A.B. Bortz, M.H. Kalos, J.L. Lebowitz, J. Comput. Phys. 17, 10 (1975).

[21] A. Das, J. Sabbatini, W.H. Żurek, Sci. Rep. 2, 352 (2012); J. Dziarmaga, J. Meisner, W.H. Żurek, Phys. Rev. Lett. 101, 115701 (2008); J. Dziarmaga, M. Tylutki, W.H. Żurek, Phys. Rev. B 84, 094528 (2011).

[22] L. Amico, D. Aghamalyan, F. Auksztol, H. Crepaz, R. Dumke, L.C. Kwek, Sci. Rep. 4, 4298 (2014) M. Sakamoto, K. Oka, R. Morita, N. Murakami, Opt. Lett. 38, 3661 (2013); A.R. Kolovsky, New J. Phys. 8, 197 (2006).

[23] M.Y. Choi, Phys. Rev. B 48, 15920 (1993). 